TPR-97-03, ULM-TP/97-5

April 1997

\title{
Semiclassical Interpretation of the Mass Asymmetry in Nuclear Fission
}

\author{
M. Brack ${ }^{1}$, S. M. Reimann ${ }^{2}$ and M. Sieber ${ }^{3}$ \\ ${ }^{1}$ Institut für Theoretische Physik, Universität Regensburg, D-93040 Regensburg, Germany \\ ${ }^{2}$ Niels Bohr Institutet, Blegdamsvej 17, DK-2100 Copenhagen Ø, Denmark \\ ${ }^{3}$ Abteilung Theoretische Physik, Universität Ulm, D-89069 Ulm, Germany
}

\begin{abstract}
We give a semiclassical interpretation of the mass asymmetry in the fission of heavy nuclei. Using only a few classical periodic orbits and a cavity model for the nuclear mean field, we reproduce the onset of left-right asymmetric shapes at the fission isomer minimum and the correct topology of the deformation energy surface in the region of the outer fission barrier. We point at the correspondence of the single-particle quantum states responsible for the asymmetry with the leading classical orbits, both lying in similar equatorial planes perpendicular to the symmetry axis of the system.
\end{abstract}

PACS numbers:

24.75.+i General properties of fission

03.65.Sq Semiclassical theories and applications.

47.20.Ky Nonlinearity (including bifurcation theory). 
One characteristic feature of the fission of many actinide nuclei is the asymmetric mass distribution of the fission fragments. The liquid drop model [1], although able to describe many aspects of the fission process qualitatively, cannot explain this mass asymmetry in heavy nuclei where the fissility parameter $x$ is close to unity [2]: the balance between the attractive surface tension and the repulsive Coulomb force favors left-right symmetric shapes and thus also the symmetric fission. An explanation for the observed asymmetry of the fragment distributions became possible with Strutinsky's shell-correction method [3] which includes the quantal shell effects stemming from the discrete spectra of the nucleons in their mean fields. One writes the total binding energy of a nucleus consisting of $N$ neutrons and $Z$ protons as

$$
\begin{aligned}
E_{t o t}(N, Z ; \text { def })= & E_{L D M}(N, Z ; \text { def }) \\
& +\delta E_{n}(N ; \text { def })+\delta E_{p}(Z ; \text { def }) .
\end{aligned}
$$

Here $E_{L D M}$ is the liquid drop model (LDM) energy; $\delta E_{n}$ and $\delta E_{p}$ are the shell-correction energies of the neutrons and protons, respectively, which are obtained in terms of the singleparticle energies of realistic shell-model potentials. All ingredients depend on the shape of the nucleus, which is described by some suitable deformation parameters, summarized in (1) by 'def'. The shell-correction approach was very successful in reproducing experimental nuclear binding energies and fission barriers [4, 5, 6] at times where purely microscopical selfconsistent calculations of Hartree-Fock type were not yet available [7].

In Fig. 1 we show a schematic fission barrier of a typical actinide nucleus, taken along the adiabatic path through the multi-dimensional deformation space. The heavy dashed line is the LDM deformation energy which leads to a spherical ground state and to equal fragment sizes. The solid line is the total energy according to Eq. (11); it contains the typical shell oscillations coming from the shell corrections $\delta E_{n}$ and $\delta E_{p}$. These lead to a deformed ground state minimum and to a higher-lying local minimum, the so-called fission isomer. (For an extended review on the physics of the 'double-humped fission barrier', see Ref. [8].) The shapes assumed hereby have axial symmetry and left-right symmetry. When the latter is relaxed, the energy is found [9, 10] to be lowered along the way over the outer barrier, starting at the fission isomer. The gain in energy persists all the way down towards the scission point, where the nucleus breaks into two fragments of unequal size. Nonaxial deformations do not change this feature; they only lead to a slight reduction of the inner barrier (see Ref. [11] for a short review of fission barrier calculations and the role of various deformations). It is important to note that the onset of the mass asymmetry takes place already at an early stage of the fission process, long before the nucleus breaks up. It is a pure quantum effect which only comes about if the shell corrections are included into the total energy. The microscopic origin of this instability has been investigated by Gustafsson, Möller, and Nilsson [12]. They found that only two specific types of single-particle states with large angular momenta along the symmetry axis are strongly sensitive to the left-right asymmetry: one of them has the maxima of its wave functions along the central waist line of the nucleus (see the upper right in Fig. 1), whereas the other has maxima along the circumferences of two equatorial planes at some distance of the center (with opposite phases on either side, see the middle right in Fig. 1). The coupling of these states through the left-right asymmetric components of the mean field leads to a decrease of one set of eigenenergies which lie below the Fermi energy, and thus to a reduction of the total binding energy when the asymmetry is switched on (see the lower right in Fig. 1).

In the following we give a very simple and transparent semiclassical interpretation of this 

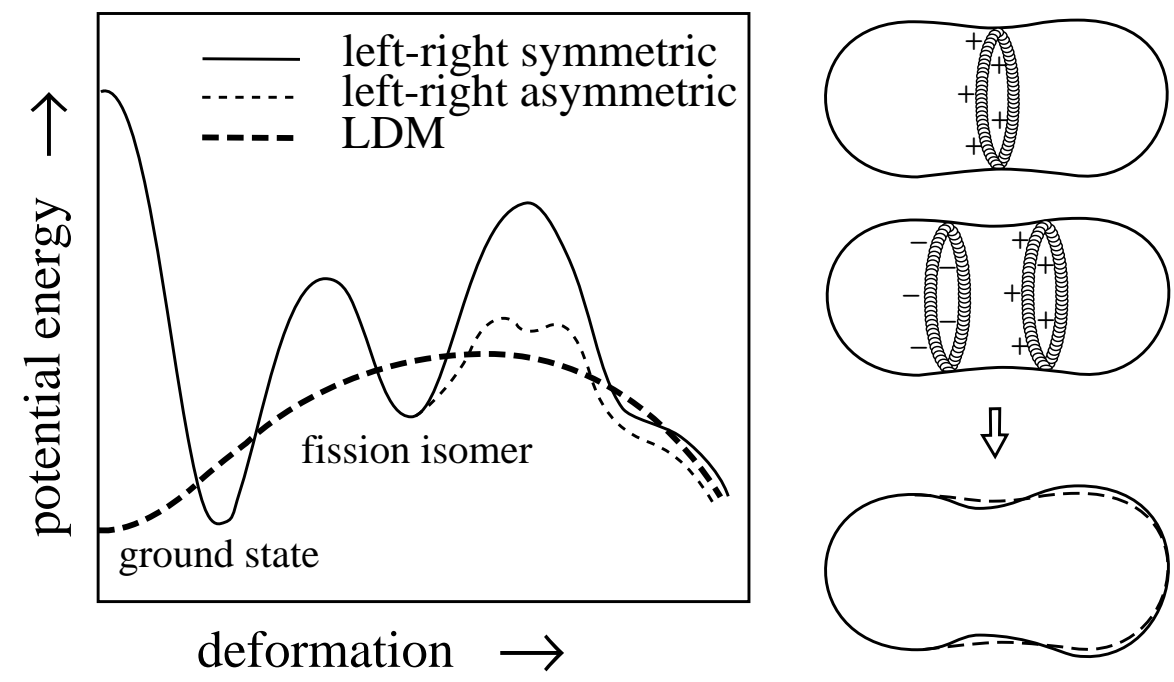

Figure 1: Left side: schematic fission barrier of a typical actinide nucleus (after Ref. [11]). Right side: schematic probability amplitudes of the leading single-particle wavefunctions responsible for the onset of mass asymmetry (after Ref. [12]).

quantum effect. We employ the periodic orbit theory (POT) developed independently by Gutzwiller 13 for systems with isolated classical orbits and by Balian and Bloch 14 for arbitrarily deformed cavities with reflecting walls. In the POT, one obtains the oscillating part of the level density of a quantum system from the so-called 'trace formula':

$$
\delta g(E) \simeq \sum_{p o} \mathcal{A}_{p o}(E) \cos \left[\frac{1}{\hbar} S_{p o}(E)-\sigma_{p o} \frac{\pi}{2}\right] .
$$

The sum is taken over all periodic orbits, labeled ' $p o$ ', of the corresponding classical system. $S_{p o}$ are the classical actions along the periodic orbits and $\sigma_{p o}$ are phases related to the number of conjugate points along the orbits. The amplitude $\mathcal{A}_{p o}$ of each orbit depends on its period, its stability and its degeneracy. Together with the smooth part $\widetilde{g}(E)$ which can be obtained in the (extended) Thomas-Fermi model, it approximates the exact quantum-mechanical level density: $\tilde{g}(E)+\delta g(E)=g(E)=\sum_{i} \delta\left(E-E_{i}\right)$, where $E_{i}$ are the eigenenergies of the system and the sum runs over all quantum states $i$. (See Ref. [15] for an introduction to the POT and detailed explanations of all the above ingredients). Gutzwiller's trace formula has initiated promising steps towards the semiclassical quantization of chaotic systems [16]. A different use of the POT [15, 17] is to obtain a coarse-grained level density by keeping only the shortest orbits with the largest amplitudes in the trace formula (2). This allows one to relate the gross-shell structure of interacting fermion systems in the mean-field approximation to a few classical orbits. Using Eq. (2), the semiclassical expression for the energy shell correction $\delta E$ becomes [17]

$$
\delta E \simeq \sum_{p o} \mathcal{A}_{p o}\left(E_{F}\right)\left(\frac{\hbar}{T_{p o}}\right)^{2} \cos \left[\frac{1}{\hbar} S_{p o}\left(E_{F}\right)-\sigma_{p o} \frac{\pi}{2}\right] .
$$

Here $E_{F}$ is the Fermi energy and $T_{p o}=d S_{p o} /\left.d E\right|_{E_{F}}$ the period of the orbit labeled $p o$. Pioneering work has been done in this direction by Strutinsky et al. [17], who generalized the Gutzwiller 
theory to systems with continuous symmetries and used it to give a semiclassical explanation of the systematics of nuclear ground-state deformations. They showed that the ground-state minima of the nuclear binding energy as functions of particle number and deformation follow the loci of constant action of the shortest periodic orbits. Another beautiful example is the beating pattern of the coarse-grained level density in a spherical cavity, which was related by Balian and Bloch [14] to the interference of the triangular and square periodic orbits, and later predicted [18] and observed [19] in metal clusters in the form of the so-called supershells.

For our semiclassical investigation, we replace the nuclear mean field by a cavity with reflecting walls and consider only one kind of particles. This should yield the main physical effects, since the neutron contribution $\delta E_{n}$ to the total energy contains the largest part of the shell oscillations, as seen, e.g., in Fig. VIII-4 of Ref. [4. We employ the parameterization $(c, h, \alpha)$ of this reference, used there to define the shape of the liquid drop. Here it defines the boundary of the cavity in cylindrical coordinates $(\rho, z, \phi)$, with the symmetry axis along $z$, by the shape function $\rho=\rho(z ; c, h, \alpha)$. $c$ is the length of the semiaxis along the $z$ direction in units of the radius $R_{0}$ of the spherical cavity $(c=1, h=\alpha=0)$. $h$ is a 'neck parameter' that regulates the formation of a neck leading to the scission of the nucleus into two fragments. $\alpha \neq 0$ yields left-right asymmetric shapes. The volume of the cavity is kept constant for all deformations. (See Ref. [4] for the details of this parameterization, and especially Figs. VII-1 and VIII-5 for the most important shapes occurring in fission.) The parameters $(c, h)$ are chosen such that the one-dimensional curve $h=\alpha=0$ along $c$ follows the adiabatic fission barrier of the LDM (shown schematically in Fig. 11). Even including the shell effects, $h=0$ gives a reasonable picture of the double-humped fission barrier.

We now have to determine the shortest periodic orbits of this system to calculate the gross-shell structure in $\delta E$. At large deformations (here $c \gtrsim 1.4$ ), these are the orbits lying in equatorial planes perpendicular to the symmetry axis 20, 21]. The positions $z_{i}$ of these planes along the $z$ axis are given by the condition that the shape function be stationary: $d \rho(z ; c, h, \alpha) /\left.d z\right|_{z_{i}}=0$. The periodic orbits have the form of regular polygons and are characterized by $(p, t)$, where $p$ is the number of reflections at the boundary and $t$ the number of windings around the symmetry axis. Both numbers are restricted by $p \geq 2$ and $t \leq p / 2$. The semiclassical contribution of such orbits to the trace formula (2) has been derived by Balian and Bloch [14]; we refer to their paper for the explicit form of the amplitudes $\mathcal{A}_{p t}$ and phases $\sigma_{p t}$. The lengths of the orbits are $L_{p t}^{(i)}=2 p R_{i} \sin (\pi t / p)$, where $R_{i}=\rho\left(z_{i} ; c, h, \alpha\right)$, so that their actions equal $S_{p t}^{(i)}\left(E_{F}\right)=\hbar k_{F} L_{p t}^{(i)}$ in terms of the Fermi wave number $k_{F}=\sqrt{2 m E_{F}} / \hbar$.

The range of validity of Eqs. (2) and (3) is, however, limited. They are correct only as long as the orbits are well separated from neighboring periodic orbits, in particular as long as the orbits are not close to a bifurcation. At a bifurcation the amplitudes $A_{p t}$ diverge and the trace formula has to be modified. Generally, bifurcations exist in different forms, but for the shapes studied here we need consider only one type of bifurcation. It occurs when the positions $z_{i}$ of several equatorial planes coincide. In the $(c, h, \alpha)$ parameterization, there are at most three such planes. One plane always exists; the other two arise at the points $\left(c_{0}, h_{0}, \alpha_{0}\right)$ where the neck formation starts. In the symmetric case $(\alpha=0)$, one plane is always located at $z_{0}=0$ and, beyond the bifurcation point, the other two are located symmetrically at $\pm z_{1}\left(\right.$ with $\left.z_{1}>0\right)$ and contain identical periodic orbits.

Near a bifurcation point, the neighboring orbits give a joint contribution to the level density. This contribution can be derived from an oscillatory integral which contains the contributions 
of all orbits of type $(p, t)$ from the different planes. The integral is of the form [14]

$$
\delta E_{p t}=\Re e \int_{-c}^{+c} d z f_{p t}(z) \exp \left\{i k_{F} L_{p t}(z)\right\},
$$

where $f_{p t}(z)$ are slowly varying analytic functions of $z$. Since the plane positions $z_{i}$ of the periodic orbits are determined by the stationary points of the length function $L_{p t}(z)=2 p \sin (\pi t / p)$. $\rho(z ; c, h, \alpha)$, a stationary phase evaluation of (4) leads back to separate contributions to Eq. (3) for each plane, with the amplitudes and phases given in [14]. In order to obtain an approximation to (团) that is valid at the bifurcation as well as far from it, we employ a uniform approximation that is appropriate for the case of three nearly coincident stationary points in a one-dimensional oscillatory integral [22]. It is expressed in terms of Pearcey's integral and its derivatives:

$$
\begin{array}{r}
\delta E_{p t}=\Re e\left\{\left[u_{4} P\left(u_{1}, u_{2}\right)+u_{5} P_{x}\left(u_{1}, u_{2}\right)\right.\right. \\
\left.\left.+u_{6} P_{y}\left(u_{1}, u_{2}\right)\right] e^{i u_{3}}\right\},
\end{array}
$$

where Pearcey's integral is defined by

$$
P(x, y)=\int_{-\infty}^{\infty} d z \exp \left[i\left(z^{4}+x z^{2}+y z\right)\right]
$$

and $P_{x}(x, y), P_{y}(x, y)$ denotes its derivatives with respect to its first and second argument, respectively. The real constants $u_{1} \ldots u_{6}$ are determined by the semiclassical amplitudes, actions and phases of the periodic orbits. If the orbits are well separated, Eq. (5) reduces to contributions to the standard trace formula (3).

When the asymmetry parameter $\alpha$ is sufficiently large, the contributions from one plane of orbits can be evaluated in the stationary phase approximation. Both in this limit and in the symmetric case $(\alpha=0)$, the result can be expressed in terms of cylindrical Bessel functions $J_{\mu}(x)$, and the formulae are analogous to those for generic saddle-node and pitchfork bifurcations [23]. We give here the result for $\delta E_{p t}$ in the symmetric case:

$$
\begin{aligned}
\delta E_{p t}= & \Re e\left[\sqrt{\frac{\pi k_{F}\left|\Delta L_{p t}\right|}{2}} e^{i\left(k_{F} \bar{L}_{p t}-3 p \pi / 2\right)}\right. \\
& \times\left\{\left(\frac{\hbar^{2} \mathcal{A}_{p t}^{(1)}}{\left[T_{p t}^{(1)}\right]^{2}}+\frac{\hbar^{2} \mathcal{A}_{p t}^{(0)}}{\sqrt{2}\left[T_{p t}^{(0)}\right]^{2}}\right)\left(\nu J_{1 / 4}\left(k_{F}\left|\Delta L_{p t}\right|\right) e^{i \pi / 8}+J_{-1 / 4}\left(k_{F}\left|\Delta L_{p t}\right|\right) e^{-i \pi / 8}\right)\right. \\
& \left.+\left(\frac{\hbar^{2} \mathcal{A}_{p t}^{(1)}}{\left[T_{p t}^{(1)}\right]^{2}}-\frac{\hbar^{2} \mathcal{A}_{p t}^{(0)}}{\sqrt{2}\left[T_{p t}^{(0)}\right]^{2}}\right)\left(J_{3 / 4}\left(k_{F}\left|\Delta L_{p t}\right|\right) e^{i 3 \pi / 8}+\nu J_{-3 / 4}\left(k_{F}\left|\Delta L_{p t}\right|\right) e^{-i 3 \pi / 8}\right)\right\} .
\end{aligned}
$$

Here $\bar{L}_{p t}=\left[L_{p t}^{(1)}+L_{p t}^{(0)}\right] / 2$ and $\Delta L_{p t}=\left[L_{p t}^{(1)}-L_{p t}^{(0)}\right] / 2$ in terms of the lengths $L_{p t}^{(0)}, L_{p t}^{(1)}$ of the orbits $p t$ situated at $z=z_{0}$ and $z= \pm z_{1}$, respectively. $\nu$ equals -1 before the bifurcation (i.e., for only one orbit plane) and +1 after the bifurcation (for three orbit planes).

In the right-hand panels of Fig. 2 we show contour plots of the semiclassical shell-correction energy $\delta E$ in the $(c, \alpha)$ plane for two values of the neck parameter. The energy unit is $E_{0}=$ $\hbar^{2} / 2 m R_{0}^{2}$, where $R_{0}$ is the radius of the spherical box. The Fermi wave number $k_{F}$ was chosen 

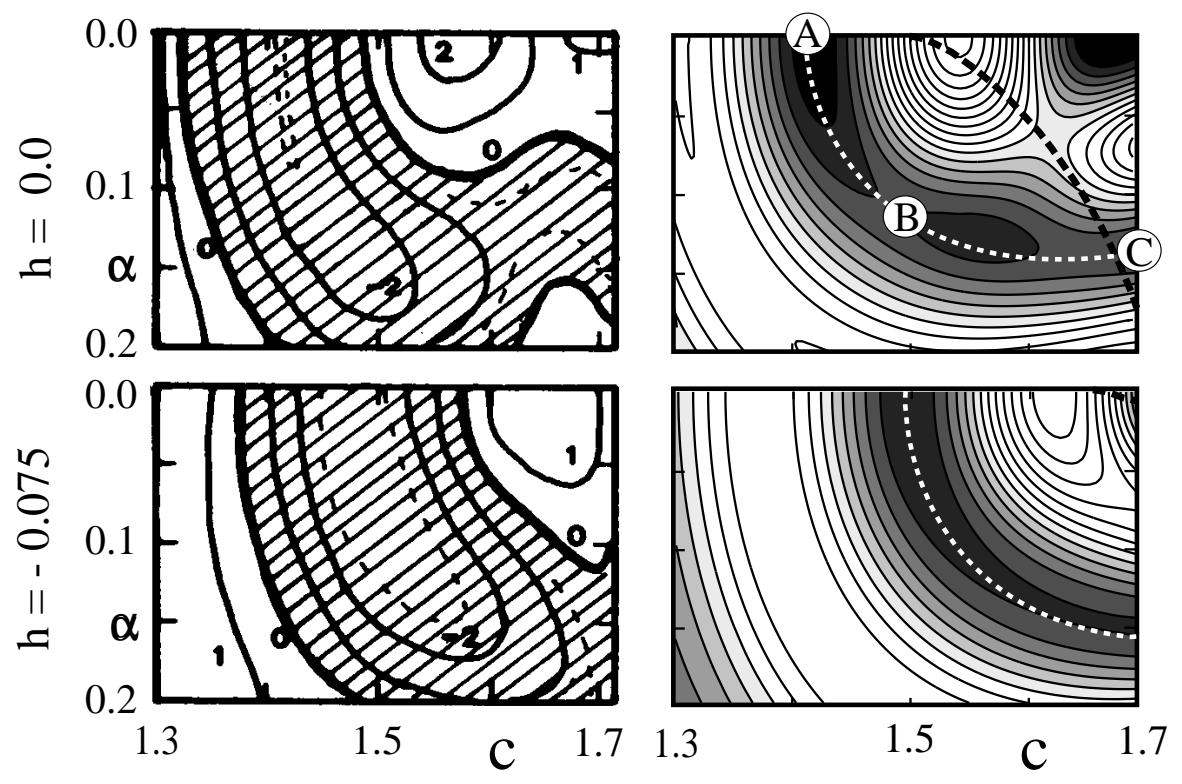

Figure 2: Contour plots of deformation energy versus elongation $c$ and asymmetry $\alpha$ for two values of the neck parameter: $h=0$ (above) and $h=-0.075$ (below). Left side: quantum-mechanical neutron shell-correction energy of ${ }^{240} \mathrm{Pu}$ from Ref. [4]. Right side: semiclassical shell-correction energy $\delta E$. White dashed lines indicate the loci of constant classical action of the central equatorial periodic orbits; black dashed lines the loci of the bifurcation points.

such that $\delta E$ has a minimum at the deformation $c=1.42, h=\alpha=0$ of the fission isomer. On the left of Fig. 2 we have reproduced the neutron shell-correction energy $\delta E_{n}$ of the nucleus ${ }^{240} \mathrm{Pu}$, obtained in Ref. [4] with a realistic Woods-Saxon type shell-model potential. We see that the semiclassical result correctly reproduces the topology of the deformation energy in the $(c, \alpha)$ plane for both values of $h$, in particular the onset of the mass asymmetry at the fission isomer. It should be noted that we have only included orbits with winding number one $(t=1)$ and with up to $p_{\max }=10$ reflections. The results for $\delta E$ are the same within a few percent when only orbits with $p=2$ and 3, i.e., only the diameter and triangle orbits, are included. The loci of the bifurcation points $\left(c_{0}, \alpha_{0}\right)$ are indicated in Fig. 2 by the black heavy dashed lines (that for $h=-0.075$ is hardly visible in the upper right corner of the plot). This shows that the essential feature, namely the energy gain due to the asymmetric deformations for $c \geq 1.42$, is brought about by only two classical orbits: the diameter and the triangle in the central equatorial plane. The white dashed lines give the loci of constant actions of the periodic orbits at $z_{0}$, fixing their value at $\alpha=0$. (Note that the actions of all orbits in a given equatorial plane have the same deformation dependence.) We see that the valley that leads from the isomer minimum over the outer fission barrier in the energetically most favorable way is following exactly the path of constant action of the leading classical orbits; the path is practically identical with that obtained in the quantum-mechanical shell-correction calculations. A more detailed comparison with the latter, together with the calculational details of our studies, will be presented in a forthcoming publication. 

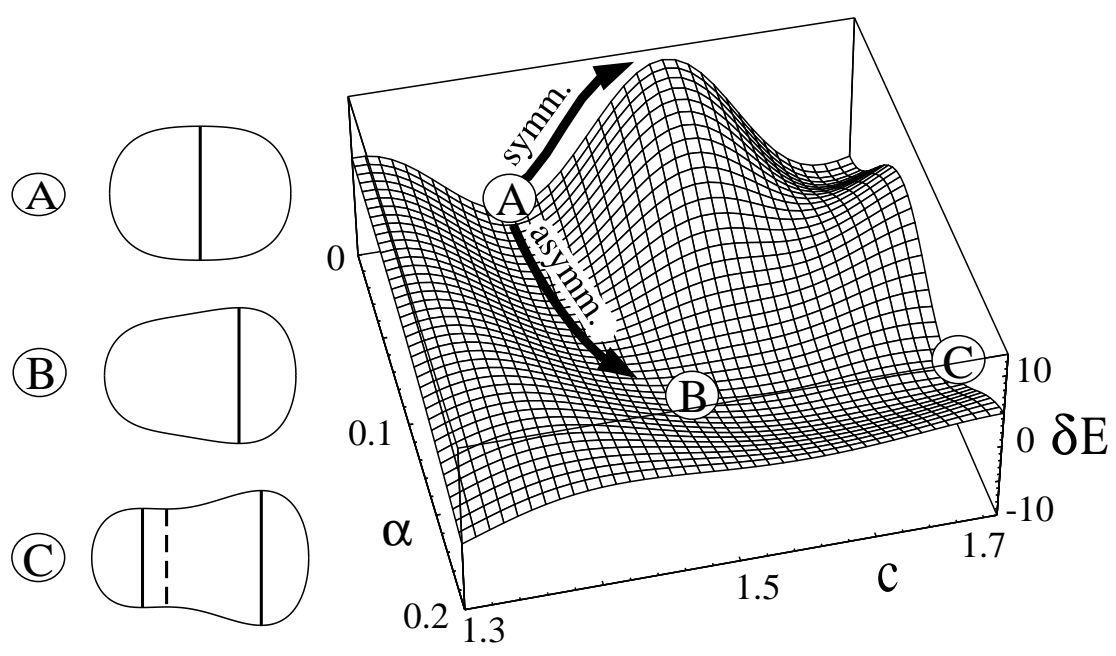

Figure 3: Right side: semiclassical shell-correction energy $\delta E$ as in Fig. 2 for $h=0$, but in a three-dimensional perspective view. Arrows 'symmetric' and 'asymmetric' show two alternative fission paths. Left side: shapes found along the asymmetric fission path; the equatorial planes of periodic orbits included in the trace formula are shown by the vertical lines (solid lines for stable and dashed lines for unstable orbits).

In Fig. 3, we show the same results as in the upper right part of Fig. 2, but in a perspective view of a three-dimensional energy surface. On the left, the shapes of the cavity are given corresponding to the points $\mathrm{A}$ at the isomer minimum and the points $\mathrm{B}$ and $\mathrm{C}$ along the asymmetric fission barrier (see also the corresponding points in Fig. 2). Note that $\mathrm{C}$ lies beyond the bifurcation point and thus contains three planes of periodic orbits.

In summary, we have shown how a specific quantum effect, causing a drastic rearrangement of the shape of a complex many body system, can be described semiclassically by the constancy of the actions of a few periodic orbits. We point out the close correspondence of the equatorial planes of these orbits (see the left of Fig. 3) with the locations of the wave function maxima (see the right of Fig. 1) of the relevant quantum states. We also note that the classical dynamics of this system is rather chaotic, particularly in the asymmetric case and for small values of the conserved angular momentum $L_{z}$ [24]. The regular regions in phase space, connected to the stable periodic orbits with axial degeneracy, are still strong enough to cause this important shell effect.

M. S. wishes to acknowledge financial support by the Deutsche Forschungsgemeinschaft under Contract No. DFG-Ste 241/6-1 and /7-2.

\section{References}

[1] N. Bohr and J. A. Wheeler, Phys. Rev. 56, 426 (1939).

[2] $x=E_{c} / 2 E_{s}$, where $E_{c}$ and $E_{s}$ are the Coulomb and surface energy, respectively, of the charged liquid drop. When $x \geq 1$, the drop is unstable against fission. For further details see, e.g., L. Wilets: Theories of nuclear fission (Clarendon Press, Oxford, 1964). 
[3] V. M. Strutinsky, Nucl. Phys. A 122, 1 (1968); and earlier references quoted therein.

[4] M. Brack, J. Damgård, A. S. Jensen, H. C. Pauli, V. M. Strutinsky, and C. Y. Wong, Rev. Mod. Phys. 44, 320 (1972).

[5] S. G. Nilsson, C. F. Tsang, A. Sobiczewski, Z. Szymański, S. Wycech, C. Gustafsson, I.-L. Lamm, P. Möller, and B. Nilsson, Nucl. Phys. A 131, 1 (1969).

[6] M. Bolsterli, E. O. Fiset, J. R. Nix, and J. L. Norton, Phys. Rev. C 5, 1050 (1972); J. R. Nix, Ann. Rev. Nucl. Sci. 22, 65 (1972).

[7] See Refs. [3, 4] for the foundation of the shell-correction method on the Hartree-Fock theory, and M. Brack and P. Quentin, Nucl. Phys. A 361, 35 (1981), for numerical tests employing realistic nucleon-nucleon interactions.

[8] S. Bjørnholm and J. E. Lynn, Rev. Mod. Phys. 52, 725 (1980).

[9] P. Möller and S. G. Nilsson, Phys. Lett. 31 B, 283 (1970).

[10] H. C. Pauli, T. Ledergerber, and M. Brack, Phys. Lett. 34B, 264 (1971).

[11] M. Brack, in: Physics and Chemistry of Fission 1979, International Symposium held in Jülich in 1979 (IAEA Vienna, 1980), Vol. I, p. 227.

[12] C. Gustafsson, P. Möller, and S. G. Nilsson, Phys. Lett. 34 B, 349 (1971).

[13] M. C. Gutzwiller, J. Math. Phys. 12, 343 (1971).

[14] R. Balian and C. Bloch, Ann. Phys. (N. Y.) 69, 76 (1972).

[15] M. Brack and R. K. Bhaduri: Semiclassical Physics (Addison and Wesley, Reading, 1997).

[16] Chaos Focus Issue on Periodic Orbit Theory, ed. P. Cvitanović: Chaos 2, 1-158 (1992).

[17] V. M. Strutinsky and A. G. Magner, Sov. J. Part. Nucl. 7, 138 (1976); V. M. Strutinsky, A. G. Magner, S. R. Ofengenden, and T. Døssing, Z. Phys. A 283, 269 (1977).

[18] H. Nishioka, K. Hansen and B. R. Mottelson, Phys. Rev. B 42, 9377 (1990).

[19] J. Pedersen, S. Bjørnholm, J. Borggreen, K. Hansen, T. P. Martin, and H. D. Rasmussen, Nature 353, 733 (1991).

[20] H. Frisk, Nucl. Phys. A 511, 309 (1990).

[21] A. G. Magner, S. N. Fedotkin, F. A. Ivanyuk, P. Meier, M. Brack, S. M. Reimann, and H. Koizumi, Ann. Phys. (Leipzig) (1997), submitted.

[22] J. N. L. Connor, Mol. Phys. 26, 1217 (1973).

[23] M. Sieber and H. Schomerus, J. Phys. A, (1997), submitted.

[24] K. Arita and K. Matsuyanagi, Prog. Theor. Phys. (Japan) 91, 723 (1994). 